\title{
Quasi-Intrinsic Angular Momentum and the Measurement of Its Spectrum
}

\author{
Roberta Zambrini and Stephen M. Barnett \\ SUPA, Department of Physics, University of Strathclyde, Glasgow G4 ONG, United Kingdom
}

(Received 24 November 2005; published 22 March 2006)

\begin{abstract}
We introduce the concept of quasi-intrinsic angular momentum to denote fields for which the mean value of the angular momentum is unaltered by a lateral shift of the rotation axis but the spectrum changes. This property is exemplified by the orbital angular momentum of a beam of light about its propagation direction. We propose an interferometric experiment to measure efficiently the exact angular momentum spectrum and variance for light beams with any arbitrary spatial distribution.
\end{abstract}

DOI: $10.1103 /$ PhysRevLett.96.113901

PACS numbers: 42.25. $-\mathrm{p}, 03.67 .-\mathrm{a}, 42.87 . \mathrm{Bg}$

Angular momentum (AM) plays an important role in the analysis of physical systems both in classical [1] and quantum mechanics [2]. A fundamental distinction between intrinsic and extrinsic AM arises when the effects of changing the rotation axis are considered. For a particle, a shift of the rotation origin changes the AM by an amount given by the AM carried by the center of mass in the new reference system. Hence the AM of a particle spinning around its stationary center is intrinsic, i.e., independent on the choice of the rotation axis, and is clearly distinguished by the extrinsic AM of a particle orbiting around one point [1].

The same effect of changing the rotation origin holds for classical or quantum fields [3]. The intrinsic and extrinsic parts of a field AM are not, however, synonymous with the spin and orbital AM. In particular, the total AM of a field in the direction of the linear momentum is always unchanged on shifting to a parallel axis, irrespective of whether it comprises spin AM, orbital AM, or a mixture of the two. In optics, for example, the Laguerre-Gaussian (LG) beams carry orbital AM $[4,5]$ that appears to be intrinsic, like any spin AM associated with circular polarization [6]. This is surprising as the orbital AM of LG beams is due to their helical phase distribution about the beam axis, the position of which coincides with a phase singularity [7]. Hence their cylindrical spatial distribution indicates a natural rotation axis and leads us to suspect that orbital AM might have an extrinsic quality. We resolve the apparent inconsistency by pointing out that the orbital AM has an intermediate and distinct character between intrinsic and extrinsic; it is quasi-intrinsic [8].

The question of the AM dependence on rotation axis is of more than purely theoretical interest because of the growing number of applications. In quantum information, for example, using orbital AM offers the possibility to access a higher dimensional state space than that provided by polarization or spin AM $[9,10]$. Retrieving the information requires us to detect the orbital AM, but such measurements are sensitive to misalignment [9-11], which again suggests an extrinsic quality. Intimately related issues are the possibility to engineer the AM spectrum seeding off-axis vortices or more complex spatial profiles in light beams [12] as well as the emergence of structures of (off-axis) vortices in many different fields [13]. In this work we analyze the extent to which the orbital AM depends on the choice of the rotation axis. As an indicator for the quasi-intrinsic character of the orbital AM we consider the variance of its $z$ component, calculated analytically for LG modes in any reference system. In view of optical applications we propose the use of a novel interferometer to measure the AM spectrum and the variance for light beams with arbitrary spatial distributions.

Theoretical analysis. - In order to avoid the complications associated with spin AM we consider a scalar field $\psi$ satisfying the equation:

$$
\left(i \alpha \partial_{z}+\partial_{x}^{2}+\partial_{y}^{2}\right) \psi=0,
$$

which models a broad class of well-known systems [14]. For $\alpha=2 k$ Eq. (1) governs the paraxial propagation of waves of wave number $k$ and is widely employed in optics [15]. For $\alpha=2 m / \hbar$ it describes the quantum dynamics of a free particle of mass $m$ in the $x y$ plane, with $z$ read as time. In both optics and quantum mechanics the $z$ component of the orbital AM is proportional to the azimuthal gradient and may be associated with the operator $\hat{\ell}_{z}=$ $-i \partial_{\phi}[2,4]$, the eigenmodes of which are characterized by helical wave profiles, like the LG beams [4,5]. As mentioned before, the AM carried by LG modes is always intrinsic in spite of the existence of a well-defined rotation axis associated with the position of their singularity [6]. Given a field with linear momentum density $\mathbf{p}$-the Poynting vector for optical fields - the AM carried by the wave in a plane $\mathbf{x}=(x, y)$ is:

$$
\mathbf{L}=\int d \mathbf{x r} \times \mathbf{p}=\int d \mathbf{x} \ell(\mathbf{x}), \quad[\mathbf{r}=(x, y, z)] .
$$

The relation between $L_{z}$ and the AM in a rotation frame centered in $\mathbf{r}_{0}$ (denoted by a prime) is [6]

$$
L_{z}^{\prime}=\int d \mathbf{x}\left[\left(\mathbf{r}-\mathbf{r}_{0}\right) \times \mathbf{p}\right]_{z}=L_{z}-x_{0} P_{y}+y_{0} P_{x} .
$$

If the total linear momentum vector points in the $z$ direc- 
tion, i.e., $\mathbf{P}_{\perp}=\left(P_{x}, P_{y}\right)=0$, then $L_{z}$ is always intrinsic and independent on the position of the phase singularity.

Let us first illustrate Eq. (3) by comparing two fields with different transverse linear momenta: $\psi_{a}$ is a LG mode with radial index $p=0$ and helical index $\ell=1$ [Fig. 1(a)], while $\psi_{b}=M \psi_{a}$ is obtained by scattering $\psi_{a}$ through a small transparent object placed off axis and described by the mask function $M(\mathbf{x})=\exp \left\{i \pi e^{-10\left[(x-1)^{2}+y^{2}\right]}\right\} \quad$ [16] [Fig. 1(b)]. Both fields have the same total $L_{z}$, but this is intrinsic for $\psi_{a}$ and extrinsic for $\psi_{b}$. This difference is due to the change of phase in the field, introduced by the scattering, that deflects slightly the momentum of $\psi_{b}$ $\left(P_{x} \neq 0\right)$. By virtue of Eq. (3), the $z$ component of the total $\mathrm{AM}$ of $\psi_{b}$ in a generic frame centered in $\mathbf{x}_{0}=\left(x_{0}, y_{0}\right)$ is $L_{z}^{\prime} / L_{z} \sim 1+65 \pi / 162 e^{5 / 3} y_{0}$. Therefore, a small transparent bead off axis does not rotate when illuminated by a LG mode [16], but makes the AM $L_{z}$ of the output beam extrinsic.

The similarity between paraxial propagation for light waves and evolution of wave functions in the Schrödinger picture, established through Eq. (1), suggests an interesting reading of Eq. (3). The total linear momentum of an optical wave $\psi[17]$ per unit length is $[4,18]$

$$
\mathbf{P}_{\perp}=\frac{\epsilon_{0}}{2 i \omega} \int \psi^{*} \nabla_{\perp} \psi d \mathbf{x} .
$$

Equivalent expressions, with different prefactors, hold for acoustic waves or quantum wave functions. Equation (4) can be read as the average of the transverse momentum operator $\hat{\mathbf{p}}_{\perp}=-i \nabla_{\perp}$. It is convenient to introduce the complex vector $|\psi\rangle$ and its dual $\langle\psi|$ with the scalar product $\langle\psi \mid \xi\rangle=\epsilon_{0} /(2 \omega) \int \psi^{*} \xi d \mathbf{x} \quad$ so that $\quad \mathbf{P}_{\perp} \rightarrow\left\langle\psi\left|\hat{\mathbf{p}}_{\perp}\right| \psi\right\rangle$. Similarly, the orbital component of the total AM $L_{z}$ in Eq. (2) can be interpreted as an average, in the quantum formalism, of the operator $\hat{\ell}_{z}=\hat{x} \hat{p}_{y}-\hat{y} \hat{p}_{x}$ :

$$
L_{z}=\int \ell_{z}(\mathbf{x}) d \mathbf{x} \rightarrow\left\langle\hat{\ell}_{z}\right\rangle
$$

with the shortened notation $\langle\hat{A}\rangle=\langle\psi|\hat{A}| \psi\rangle$. It is natural to introduce the quantity $\mathcal{P}_{\ell}(z)=\int\left|a_{\ell}(r, z)\right|^{2} r d r$, in each $z$ plane, with $\psi(r, \phi, z)=\gamma \sum_{\ell} a_{\ell}(r, z) \exp (i \ell \phi)$ and $\gamma$ chosen such that $\sum_{\ell} \mathcal{P}_{\ell}=1$. In quantum theory $\mathcal{P}_{\ell}$ is an
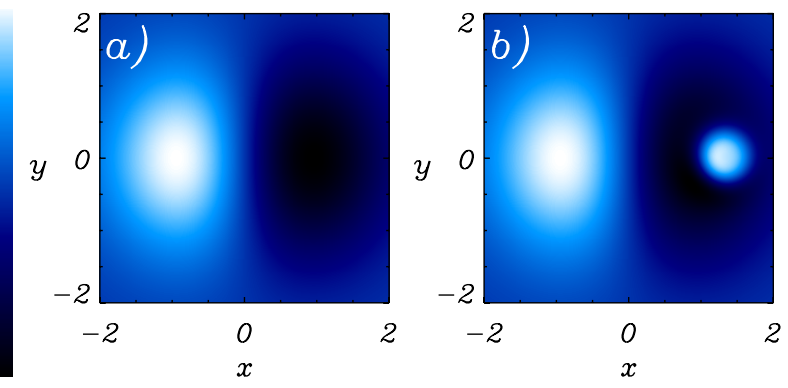

FIG. 1 (color online). Real part of $\psi_{a}(\mathbf{x})$, (a), and $\psi_{b}(\mathbf{x}),(\mathrm{b})$. Both beams have the same "doughnut" intensity profile and the same AM, but for $\psi_{b} L_{z}$ is not intrinsic.
AM probability and in classical optics it is a fraction of the total intensity. Then the average orbital AM is $L_{z} \propto$ $\sum_{\ell} \mathcal{P}_{\ell} \ell=\bar{\ell}$. Within this formalism Eq. (3) can be reformulated as $\left\langle\hat{\ell}_{z}^{\prime}\right\rangle=\left\langle\hat{\ell}_{z}\right\rangle-x_{0}\left\langle\hat{p}_{y}\right\rangle+y_{0}\left\langle\hat{p}_{x}\right\rangle$-this establishes that it is the average orbital AM in the beam direction that is intrinsic.

There are clearly some difficulties arising in considering the orbital AM as truly intrinsic. A preferred rotation axis is indeed naturally identified when considering LG beams. This suggests that the observation that the average AM is intrinsic in the linear momentum direction does not always capture all the relevant information. The density of AM, in particular, is significantly affected by a shift in reference frame [19], as shown by the examples in Fig. 2. Equivalently, the decomposition into orbital AM eigenstates and the spectrum $\mathcal{P}_{\ell}$ also changes with the reference frame. Further insight into the intrinsic or extrinsic nature of orbital AM is provided by considering the dimensionless variance, defined as

$$
V=\frac{\left\langle\psi\left|\hat{\ell}_{z}^{2}\right| \psi\right\rangle}{\langle\psi \mid \psi\rangle}-\frac{\left\langle\psi\left|\hat{\ell}_{z}\right| \psi\right\rangle^{2}}{\langle\psi \mid \psi\rangle^{2}}=\sum_{\ell=-\infty}^{+\infty} \mathcal{P}_{\ell}(\ell-\bar{\ell})^{2} .
$$

This variance is clearly vanishing for a beam with helical phase $\psi(\mathbf{r})=R(r, z) \exp (i \ell \phi)$, such as the LG beam $\psi_{a}$. It is clear, however, that any mode that is not of this form will have a nonzero $\mathcal{V}$. The variance of $\psi_{b}$, for example, is $\approx$ 2.4.

In a shifted reference frame the square of the AM operator is $\left(\hat{\ell}_{z}^{\prime}\right)^{2}=\hat{\ell}_{z}^{2}-\hat{\ell}_{z} \hat{m}_{z}-\hat{m}_{z} \hat{\ell}_{z}+\hat{m}_{z}^{2}$ where we have defined $\hat{m}_{z}=x_{0} \hat{p}_{y}-y_{0} \hat{p}_{x}$. It follows that the variances $V^{\prime}$ and $V$ are related by

$$
\begin{aligned}
V^{\prime}= & V+\left\langle\hat{m}_{z}^{2}\right\rangle-\left\langle\hat{m}_{z}\right\rangle^{2}-2\left(\left\langle\hat{\ell}_{z} \hat{m}_{z}\right\rangle-\left\langle\hat{\ell}_{z}\right\rangle\left\langle\hat{m}_{z}\right\rangle\right) \\
& -i\left(x_{0}\left\langle\hat{p}_{x}\right\rangle-y_{0}\left\langle\hat{p}_{y}\right\rangle\right),
\end{aligned}
$$

where we have introduced normalized waves $\psi / \sqrt{\langle\psi \mid \psi\rangle}$. We stress that Eq. (7) is a general result for the AM variance, valid in quantum mechanics and for any scalar field $\psi$.

Even if the mean momentum points in the $z$ direction $\left(\left\langle\hat{p}_{x}\right\rangle=\left\langle\hat{p}_{y}\right\rangle=0\right)$ the variance (7) will still depend on the
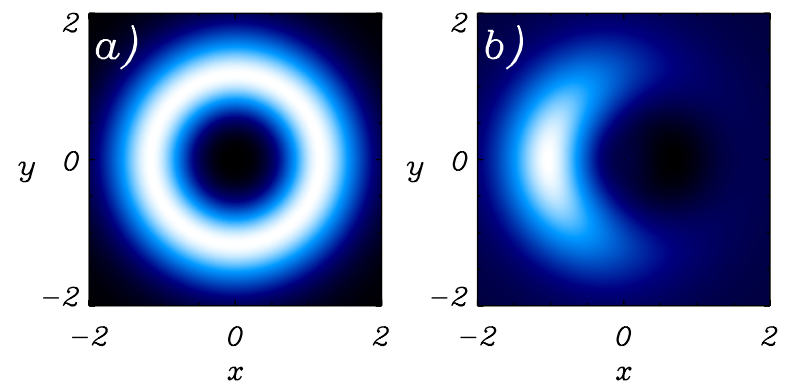

FIG. 2 (color online). (a) AM density $\ell_{z}(\mathbf{x})$ for the beam $\psi_{a}$ about the origin. This corresponds to the LG mode spatial intensity distribution. (b) $\ell_{z}^{\prime}$ about a shifted axis passing from $(0,1,0)$. 
axis about which the AM is calculated, being:

$$
\mathcal{V}^{\prime}=\mathcal{V}-2\left\langle\hat{\ell}_{z} \hat{m}_{z}\right\rangle+\left\langle\hat{m}_{z}^{2}\right\rangle .
$$

This dependence on the rotation axis, even if parallel to the linear momentum vector, constitutes a fundamental difference with respect to the average AM, Eq. (3). Let us analyze the relevance of the last two terms in Eq. (8). The average $\left\langle\hat{\ell}_{z} \hat{m}_{z}\right\rangle$ vanishes for any mode, like LG modes, with a symmetry $\psi(x, y)= \pm \psi(-x,-y)$. However, the last term $\left\langle\hat{m}_{z}^{2}\right\rangle$ cannot be zero for any shift $\left(x_{0}, y_{0}\right)$, unless the mode is a plane wave. We conclude that the AM variance will always depend on the axis about which it is calculated: $\mathcal{V}^{\prime} \neq \mathcal{V}$, as $\left\langle\hat{m}_{z}^{2}\right\rangle$ will be different from zero, even if $\left\langle\hat{m}_{z}\right\rangle=0$.

For any LG mode Eq. (8) reduces to $\mathcal{V}^{\prime}=r_{0}^{2}\left\langle\hat{p}_{x}^{2}\right\rangle$, which increases quadratically with the axis shift $r_{0}=\sqrt{x_{0}^{2}+y_{0}^{2}}$. The transverse second derivative can be replaced with the longitudinal one in virtue of the paraxial Eq. (1), leading to $\mathcal{V}^{\prime}=i r_{0}^{2}\left\langle\partial_{z}\right\rangle$, a quantity that can be evaluated analytically for any LG mode [20]:

$$
\mathcal{V}^{\prime}=r_{0}^{2} \frac{k}{2 z_{R}}(2 p+|\ell|+1) .
$$

The dependence on the indices $p$ and $\ell$ is reminiscent of the energy value of the LG eigenmodes for the harmonic oscillator and we see that higher order modes are more sensitive to displacements. The prefactor is the square of the shift $r_{0}$ scaled with the beam waist at the focal plane $w=\sqrt{2 z_{R} / k}$. Interestingly, any paraxial wave satisfying, for symmetry reasons, the condition $\left\langle\hat{p}_{x}^{2}\right\rangle=\left\langle\hat{p}_{y}^{2}\right\rangle$ has an associated variance $\mathcal{V}^{\prime}$ that is independent on the distance $z$. Therefore, even if away from the focal plane the shift $r_{0}$ will become negligible with respect to the beam spread, the variance $\mathcal{V}^{\prime}$ is unchanged in any plane. We note that in the limit of $r_{0} \ll w$ and in general for large Rayleigh range $z_{R}$ (plane wave limit), the variance of the displaced mode (9) tends to vanish as expected. For Gaussian beams $\ell=$ $p=0$ Eq. (9) follows immediately from the uncertainty principle with $\left\langle\hat{p}_{x}^{2}\right\rangle=1 / 4\left\langle\hat{x}^{2}\right\rangle$ and $\left\langle\hat{x}^{2}\right\rangle=w^{2} / 4$. As the analytical expression (9) for LG modes makes manifest, the variance $\mathcal{V}$ is an indicator of the relevance of the choice of the rotation axis position within the beam spatial distribution even when the average AM is unchanged. This measurable effect of a shift in the rotation axis suggests that orbital AM is more appropriately characterized as quasi-intrinsic.

Experimental device. - In the following we propose a device to measure directly the variance (6) as well as the angular spectrum of any field in the context of optics. Let us consider an interferometer with a pair of Dove prisms in its arms, azimuthally rotated to a relative angle $\theta$, as represented in Fig. 3 [21].

The intensity difference of the two output beams, normalized for convenience with the input intensity, provides the real part of the function of the angle:

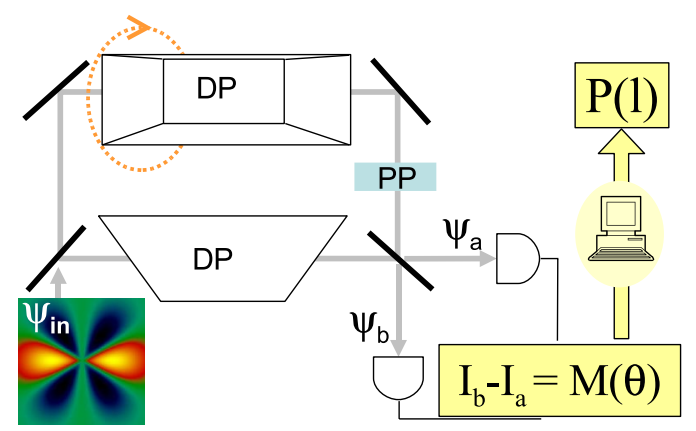

FIG. 3 (color online). Interferometer containing two Dove prisms (DP) and a phase plate (PP). The difference of the intensities outputs measured with and without the PP gives $M(\theta) . \mathcal{P}_{\ell}$ is calculated for any input $\psi_{\text {in }}$, Eq. (11).

$$
M(\theta)=\int d \mathbf{x} \psi^{*} \exp \left(i \theta \hat{\ell}_{z}\right) \psi / \int d \mathbf{x}|\psi|^{2} .
$$

It can be recognized that Eq. (10) is the average of the rotation operator generated by $\hat{\ell}_{z}$ and therefore we expect $M$ to be a complex quantity. The addition of a phase plate, introducing a phase shift of $\pi / 2$ between the beams in the interferometer, allows us to measure the imaginary part of $M(\theta)$. The complex quantity $M(\theta)$ is important, not only for its relation with the rotation operator, but also because it is related to the probabilities $\mathcal{P}_{\ell}$ simply by a Fourier transform

$$
\mathcal{P}_{\ell}=\frac{1}{2 \pi} \int d \theta M(\theta) \exp (-i \theta \ell) .
$$

Therefore the device represented in Fig. 3 can be used to measure the AM spectrum through the distribution $\mathcal{P}_{\ell}$. A priori knowledge of the symmetry $\mathcal{P}_{\ell}=\mathcal{P}_{-\ell}$ in the spectrum allows the use of the interferometer without phase plate. The novelty of this device, when compared to holographic measurements, lies in the fact that the measurement spans the angle space and provides the exact $\mathcal{P}_{\ell}$ - via a Fourier transform - for any spatial profile of the input beam. We note that the phase measurement implied in our interferometer can be turned into a frequency one by rotating the Dove prism at constant angular velocity during the experiment. The measured frequencies would than reflect the rotational Doppler shift induced by this motion [5]. It has previously been demonstrated that this shift can be used as a method to determine the spectrum of AM [22]. In contrast with other methods, however, our interferometer has the advantage of ease of operation together with the fact that it depends neither on the purity of the measured state nor on the distribution in higher radial index values $p$.

A second important use of this interferometer is to access directly the variance $\mathcal{V}$. In the limit of small relative angle $\theta$ the intensity difference at the output of the interferometer leads to both the expectation value of the AM (by using the phase plate) and its second order moment (without the phase plate). The exact evaluation of the 
variance would clearly need us to measure the interferometer outputs for all the angles $\theta$, so that the complete distribution $\mathcal{P}_{\ell}$ is found. Nevertheless, by measuring $M(\theta)$ for small angles, it would be possible to access the variance $\mathcal{V} \approx\left\{1-\operatorname{Re}[M(\theta)]-\operatorname{Im}^{2}[M(\theta)]\right\} / \theta^{2}$, with limited experimental effort.

Discussion and conclusions. - The quasi-intrinsic character of the orbital AM is ultimately related to the dependence of its density on the rotation axis through $\mathbf{r}$ in Eq. (2) $[11,19]$. This contrasts strongly with the purely intrinsic character of any spin $\mathrm{AM} \epsilon_{0} /(2 i \omega) \int d \mathbf{x} E_{x}^{*} E_{y}-E_{x} E_{y}^{*}$ associated with the elliptical polarization of an optical beam. For this quantity the integrand is independent of any choice of rotation axis and it necessarily follows that the mean and variance of the total spin AM will also be independent of this choice. In this way we see that spin AM is properly characterized as intrinsic and the quasi-intrinsic character is a specific property of the orbital AM. Indeed, the orbital AM uncertainty created by misaligned measurements is a fundamental feature to be exploited to guarantee the security in optical communications [10] and cryptography.

We stress that $\mathcal{V}$ is different from the integral of the squared AM density $\int d \mathbf{x} \ell_{z}^{2}(\mathbf{x})=-\frac{\epsilon_{0}^{2}}{4 \omega^{2}} \frac{1}{4} \int d \mathbf{x}\left(\psi^{*} \frac{\partial \psi}{\partial \phi}-\right.$ c.c. $)^{2}$, which is nonlinear in the intensity. The variance (6) is the natural choice because it is accessible to experiments and it has a clear meaning in quantum mechanics.

In conclusion, we have demonstrated the distinctive quasi-intrinsic character of the orbital component of AM and suggested as an indicator of this the dependence of the variance (6) on the rotation axis. This property adds security to communications and cryptography based on orbital, instead of spin, AM. The variance for LG modes has been evaluated and may serve as a tool to characterize any misalignment effects in the detection of these beams. We have proposed an interferometer spanning the angle space to measure the orbital AM spectrum of any light beams and we have shown the possibility to access the variance $\mathcal{V}$ with minor experimental effort. The main improvement compared with other devices will be the possibility to easily access the exact AM spectrum for arbitrary spatial distribution of the analyzed beam.

We stress that our analysis is valid in general for any $\mathrm{AM}$ in quantum mechanics or for scalar fields. Many physical systems including nonlinear optics, acoustics, Bose-Einstein condensates, plasmas, and fluids show the emergence of arrays of off-axis vortices often as result of the instability of an higher order AM eigenstate $[5,13]$. These off-axis vortices clearly have a large variance $\mathcal{V}$ and it would be interesting to understand if this is connected to the minimization of the energy of the spontaneous structure.

We thank Miles Padgett for helpful suggestions. This work was supported by the UK Engineering and Physical Sciences Research Council (GR/S03898/01).
[1] H. Goldstein, C.P. Poole, and J.L. Safko, Classical Mechanics (Addison Wesley, Reading, MA, 2002), 3rd ed.

[2] A.R. Edmonds, Angular Momentum in Quantum Mechanics (Princeton University Press, Princeton, NJ, 1957).

[3] S. Weinberg, Gravitation and Cosmology: Principles and Applications of the General Theory of Relativity (Wiley, New York, 1972), p. 47.

[4] L. Allen, M. W. Beijersbergen, R. J. C. Spreeuw, and J. P. Woerdman, Phys. Rev. A 45, 8185 (1992).

[5] L. Allen, S. M. Barnett, and M. J. Padgett, Optical Angular Momentum (Institute of Physics Publishing, Bristol, 2003).

[6] M. Berry, Singular Optics, edited by M. Soskin and M. V. Vasnetsov, Proceedings of the SPIE-International Society for Optical Engineering Vol. 3487 (SPIE, Bellingham, WA, 1998), p. 6; reprinted in [5].

[7] J.F. Nye and M. V. Berry, Proc. R. Soc. London Ser. A 336, 165 (1974).

[8] This expression was suggested by L. Allen during the conference "Physics-A Century after Einstein," Warwick, UK, 10-14 April 2005.

[9] A. Mair, A. Vaziri, G. Weihs, and A. Zeilinger, Nature (London) 412, 313 (2001).

[10] G. Gibson, J. Courtial, M. J. Padgett, M. Vasnetsov, V. Pas'ko, S. M. Barnett, and S. Franke-Arnold, Opt. Express 12, 5448 (2004).

[11] A. Vaziri, G. Weihs, and A. Zeilinger, J. Opt. B 4, S47 (2002); M. V. Vasnetsov, V. A. Pas'ko, and M. S. Soskin, New J. Phys. 7, 46 (2005).

[12] G. Molina-Terriza, J. P. Torres, and L. Torner, Phys. Rev. Lett. 88, 013601 (2002); S. S. R. Oemrawsingh, E. R. Eliel, G. Nienhuis, and J. P. Woerdman, J. Opt. Soc. Am. A 21, 2089 (2004); L. Torner, J. P. Torres, and S. Carrasco, Opt. Express 13, 873 (2005).

[13] L. Pitaevskii and S. Stringari, Bose-Einstein Condensation (Clarendon, Oxford, 2003); L. M. Pismen, Vortices in Nonlinear Fields (Oxford University Press, New York, 1999); R. Marchiano and J.-L. Thomas, Phys. Rev. E 71, 066616 (2005), and references therein.

[14] D. Marcuse, Light Transmission Optics (Van Nostrand Reinhold, New York, 1972).

[15] A. E. Siegman, Lasers (University Science Books, Mill Valley, 1986).

[16] R. Zambrini and S. M. Barnett, J. Mod. Opt. 52, 1045 (2005).

[17] For definiteness we can assume that $E_{x}=\psi(\mathbf{x}) \exp (i k z-$ $i \omega t)$ [18].

[18] S. J. van Enk and G. Nienhuis, Opt. Commun. 94, 147 (1992).

[19] A. T. O. Neil, I. MacVicar, L. Allen, and M. J. Padgett, Phys. Rev. Lett. 88, 053601 (2002).

[20] See Eq. (22) in: H. Buchholz, The Confluent Hypergeometric Function (Springer-Verlag, Berlin, 1969), p. 144.

[21] J. Leach, M. J. Padgett, S. M. Barnett, S. Franke-Arnold, and J. Courtial, Phys. Rev. Lett. 88, 257901 (2002); S. M. Barnett and R. Zambrini, J. Mod. Opt. 53, 613 (2006).

[22] M. V. Vasnetsov, J. P. Torres, D. V. Petrov, and L. Torner, Opt. Lett. 28, 2285 (2003). 